\title{
Biased feedback in brain-computer interfaces
}

\author{
Álvaro Barbero ${ }^{1,2^{*}}$, Moritz Grosse-Wentrup ${ }^{2}$
}

\begin{abstract}
Even though feedback is considered to play an important role in learning how to operate a brain-computer interface $(\mathrm{BCl})$, to date no significant influence of feedback design on $\mathrm{BCl}$-performance has been reported in literature. In this work, we adapt a standard motor-imagery BCl-paradigm to study how $\mathrm{BCl}$-performance is affected by biasing the belief subjects have on their level of control over the $\mathrm{BCI}$ system. Our findings indicate that subjects already capable of operating a $\mathrm{BCl}$ are impeded by inaccurate feedback, while subjects normally performing on or close to chance level may actually benefit from an incorrect belief on their performance level. Our results imply that optimal feedback design in BCls should take into account a subject's current skill level.
\end{abstract}

\section{Findings}

Brain-computer interfaces (BCIs) enable subjects to communicate without using the peripheral nervous system by recording brain signals and translating these into control commands [1]. To operate a BCI, subjects need to learn how to intentionally modulate certain characteristics of their brain signals in order to express their intention. For example, in motor imagery, one of the most frequently used experimental paradigms in BCIs [2], subjects are instructed to haptically imagine movements of either the left or right hand, which typically induces a decrease in power of the electromagnetic field of the brain over contralateral sensorimotor cortex in the $\mu$ - and $\beta$-frequency ranges (roughly $10-14 \mathrm{~Hz}$ and $20-30 \mathrm{~Hz}$, respectively) [3]. The observed lateralization of this sensorimotor-rhythm (SMR) can then be used to infer a subject's intention.

As in any form of skill acquisition, subjects require feedback on their performance in order to learn how to optimally regulate their brain signals. While the importance of feedback in BCIs has long been recognized [1], surprisingly little is known on how feedback should be designed in BCIs in order to facilitate the skill acquisition process. In [4], the authors investigated whether instantaneous or delayed feedback proved to be more beneficial. While individual differences could be found, on average no significant effect was observed. Recently, the influence of realistic vs. abstract feedback on BCI

\footnotetext{
* Correspondence: alvaro.barbero@uam.es

'Universidad Autónoma de Madrid (Departamento de Ingeniería Informática) and Instituto de Ingeniería del Conocimiento, Francisco Tomás y Valiente 11, 28049, Madrid, Spain
}

(c) 2010 Barbero and Grosse-Wentrup; licensee BioMed Central Ltd. This is an Open Access article distributed under the terms of the Creative Commons Attribution License (http://creativecommons.org/licenses/by/2.0), which permits unrestricted use, distribution, and reproduction in any medium, provided the original work is properly cited. performance was investigated [5]. However, the authors the type of feedback on BCI performance. As such, it appears that the specfic feedback design has little influence on BCI performance.

It should be noted, however, that in previous studies only accurate feedback was considered. While it is generally accepted that feedback in skill acquisition should be timely and precise, motivation is also known to play an important role in BCIs (cf. [6]). Accordingly, subjects may benefit from feedback that trades feedback accuracy for motivation, e.g., by artificially biasing the belief subjects have on their success in the skill acquisition process.

In this work, we investigate the influence of such a feedback bias on BCI performance. Subjects participated in a standard BCI experiment, in which they were asked to navigate a falling ball into a basket in either the left or right corner of the screen by performing haptic motor imagery of either the left or right hand. A depiction of the visual interface is shown in Figure 1. Each experimental trial lasted four seconds, and was considered successful if the ball ended up in the correct halfside of the screen. While usually the horizontal position of the ball on the screen reflects the belief of the BCI system on a subject's intention, we artificially distorted this feedback. Specifically, every two milliseconds we coded the classifier's belief on a subject's intention as a value in the range [0-1]. Then, we drew a sample from a Gaussian distribution, and added this to the classifier's belief. The mean of this sample was chosen as a function of the type of bias, and its variance was determined 


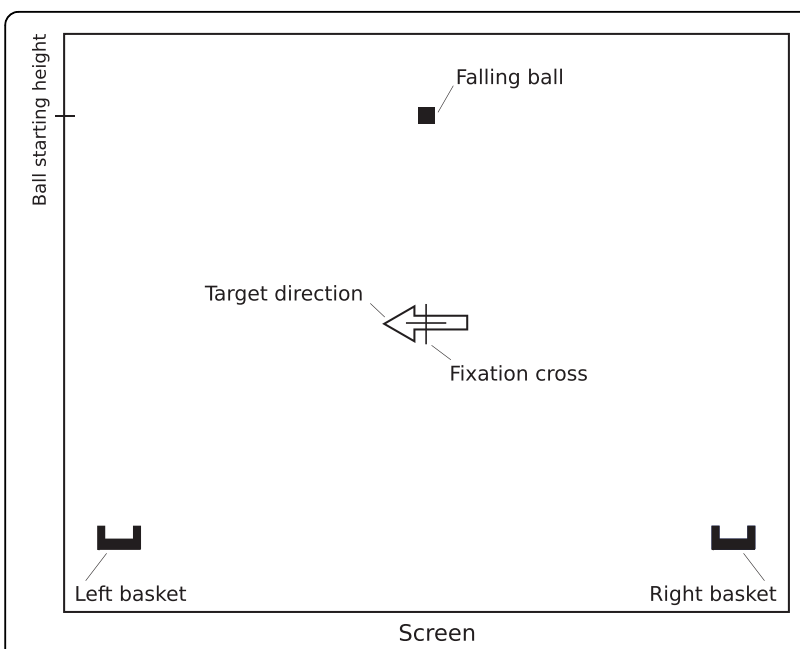

Figure 1 Setup of visual feedback. Arrangement of the elements present in the visual feedback interface of the used $\mathrm{BCl}$ system. The subject is told to look at the fixation cross, which is always present on the screen. During each trial an arrow showing the objective basket appears on screen. The position of the baskets is fixed, and the falling ball always starts at the shown position at the beginning of each trial.

heuristically and identical for all type of feedback to prevent subjects' awareness of the feedback bias $\left(\sigma^{2}=3 \cdot 10^{-4}\right)$. If the resulting value was found to be larger/smaller than the current horizontal position of the ball $(0 / 1$ representing the left/right border of the screen), the ball was was moved one step (0.003 times the width of the screen) to the right/left. At the beginning of each trial, we pseudo-randomly chose one of five means for this random distortion, such that without any meaningful BCI control by the subject the falling ball would on average end up in 1.) the intended corner of the screen (strong positive bias), 2.) half-way between the center of the screen and the intended corner (weak positive bias), 3.) in the center of the screen (no bias), 4.) halfway between the center of the screen and the incorrect corner (weak negative bias), or 5.) in the incorrect corner (strong negative bias). As such, in $80 \%$ of the trials we biased the belief the subject had on her/his performance in either a positive or negative manner, while in the remaining $20 \%$ of trials subjects received accurate feedback.

Eleven healthy subjects with a mean age of $26.18 \pm$ 4.14 years, seven of them male and four female, participated in the study, all except one were naive to BCIs. Every subject initially performed one session. Four subjects attaining a good level of BCI-control were asked to perform two additional sessions each, as we expected effects to be most prominent in well-performing subjects. Each session consisted of nine runs, with each run being composed of 15 trials per condition in pseudo- randomized order. The first three runs of each session, during which no feedback was presented to the subject, were used to train the classification system. During the following six runs, biased feedback was presented as discussed above. For each session, this resulted in a total of 36 trials for each of the five feedback biases. Mean classification accuracy was then computed for each session and feedback bias, using the undistorted classifier output hidden from the subject. Subjects were not informed that the presented feedback was biased until they had completed their last session.

The BCI system employed in this study is described in detail in [7]. Briey, classification was performed by logistic regression with $l_{1}$-regularization, using logarithmic bandpower in frequency bands ranging from 7 to $40 \mathrm{~Hz}$. Before bandpower computation, the 128-channel EEG data was spatially filtered using beamforming [7] (subjects 1 to 7 and 11) or Common Spatial Patterns (CSP) [8] (subjects 8 to 10 ).

Mean classification accuracies across all subjects and sessions are shown in Table 1 . While subject-specific effects of feedback bias could be observed (not shown here), mean classification accuracy was found to be around $68 \%$ for each type of feedback bias. In agreement with previous studies, this appears to indicate that the specific type of feedback had no general effect on BCI performance. However, Figure 2 shows the change in classification accuracy within a session due to each type of bias relative to the no-bias condition, with each dot representing one session and different subjects coded by number. Interestingly, for each type of feedback bias a negative correlation between unbiased classification accuracy and change in classification accuracy due to the bias could be observed. This correlation was found to be highly significant for a strong positive or negative bias $\left(p_{++}=0.0045, p_{-}=0.0057\right)$, and only close to or weakly significant for a weak positive or negative bias $\left(p_{+}=0.0762, p_{-}=0.0384\right)$. All $p$-values were computed by random permutation analysis with 10,000 permutations and $n=648$. Furthermore, the points of intersection of the regression lines in Figure 2 with zero change in classification accuracy roughly coincide with the unbiased classification accuracy required to reject

Table 1 Mean classification results

\begin{tabular}{cc}
\hline Feedback bias & Classification accuracy \\
\hline Strong positive bias (++) & $68.06 \%$ \\
\hline Weak positive bias (+) & $67.44 \%$ \\
\hline No bias & $68.21 \%$ \\
\hline Weak negative bias (-) & $67.90 \%$ \\
\hline Strong negative bias (- $)$ & $66.82 \%$ \\
\hline
\end{tabular}

Mean classification accuracies across all subjects and sessions for each type of feedback bias. 


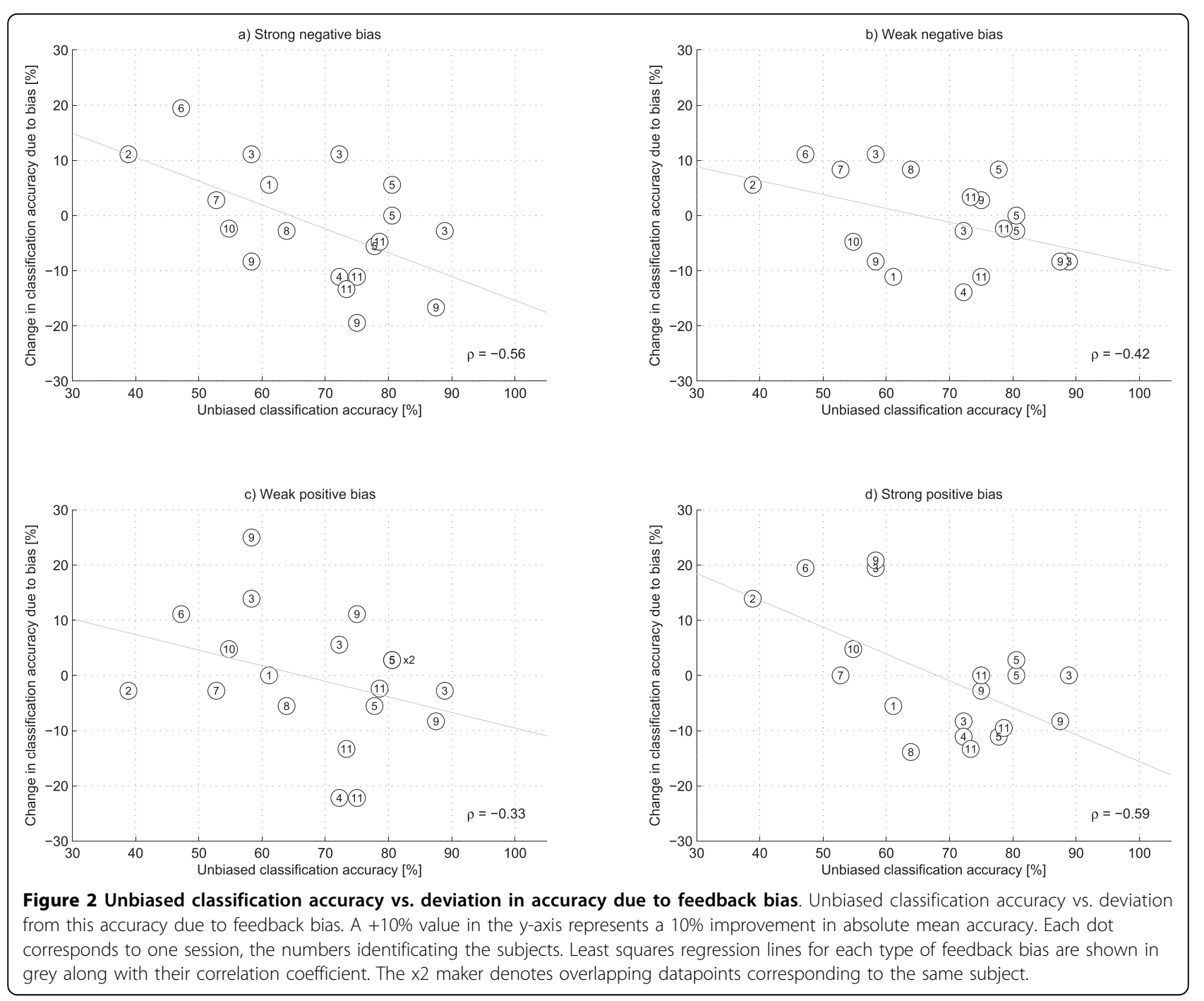

chance-level classification accuracy (for each session, an accuracy of $63.9 \%$ is required to reject the null-hypothesis of chance-level classification accuracy at significance level $\alpha=0.05)$. Our results hence appear to indicate that capable subjects, i.e., those with good classification accuracy without feedback bias, performed worse when given inaccurate feedback. Incapable subjects on the other hand, i.e., those that performed around chancelevel, appeared to benefit from a feedback distortion. While it is not surprising that inaccurate feedback decreases performance for able subjects, an increase in classification accuracy due to a feedback bias in bad-performing subjects appears counterintuitive. To further probe this result, we computed mean classification accuracies with and without feedback-bias across all sessions for which the regression analysis suggested a beneficial effect of feedback bias, i.e, for sessions on the left hand side of the intersection of the regression line with zero-change in classification accuracy in Figure 2. This resulted in mean classification accuracies of $54.41 \%$ for the unbiased case, and $58.98 \%, 56.94 \%, 59.87 \%$, and $61.78 \%$ for a strong negative, a weak negative, a weak postitive, and a strong positive bias, respectively $(n=$ 256 for each type of bias). Using a binomial distribution, these classification accuracies were found to be sufficient for rejecting the null-hypothesis of chance-level performance at significance level $\alpha=0.05$ for a strong positive bias ( $p=0.0003)$ as well as for a weak positive and strong negative bias $(p=0.0035$ and $p=0.0120$, respectively), but not for the unbiased case ( $p=0.3761)$ and a weak negative bias $(p=0.0713)$ (Bonferroni correction for multiple comparisons).

As the study design required trials with different types of feedback to be interleaved as well as subjects remaining ignorant of the feedback distortion, we could not ask subjects to report their experiences regarding different types of feedback. As such, any interpretation of the observed effects currently remains speculative. We 
hypothesize that subjects already capable of utilizing a $\mathrm{BCI}$ for means of communication are able to make use of instantaneous and accurate feedback in order to optimally regulate their SMR. In these subjects, any type of feedback bias appears to interfere with this feedback loop and hence leads to degraded performance. Accurate feedback in incapable subjects, on the other hand, may be perceived as random noise, as the horizontal movement of the falling ball is uncorrelated with the intended movement direction. We hypothesize that this perceived lack of control leads to frustration and demotivation, impeding an effective skill acquisition process. In these subjects, biased feedback may reduce the perceived randomness of the visual feedback. Specifically, our results indicate that a strong positive bias may be particularly helpful for focussing on the intended task.

In terms of feedback design for future BCI systems, our results suggest that a subject's current skill level should be taken into account. Subjects already capable of modulating their sensorimotor rhythm to some extent should receive accurate feedback. Subjects not yet capable of utilizing a $\mathrm{BCI}$, on the other hand, may benefit by designs that aim to induce a beneficial state-of-mind. While further investigations into the behavioral and neural correlates of a beneficial state-of-mind for BCIs are required (cf. $[9,10]$ for two recent studies on this topic), the results presented here suggest that incapable subjects may particularly benefit if their belief on the level of control over the BCI-system is positively biased.

\footnotetext{
Acknowledgements

This work was developed at the Max Planck Institute for Biological Cybernetics, under partial support of Spain's TIN 2007-66862 and "Cátedra UAM-IIC en Modelado y Predicción". The first author is supported by the FPU-MEC grant reference AP2006-02285. We would like to acknowledge the support of Bernd Battes for participating in the preparation and execution of the $\mathrm{BCl}$ experiments.
}

\footnotetext{
Author details

'Universidad Autónoma de Madrid (Departamento de Ingeniería Informática) and Instituto de Ingeniería del Conocimiento, Francisco Tomás y Valiente 11, 28049, Madrid, Spain. ${ }^{2}$ Max Planck Institute for Biological Cybernetics, Spemannstr. 38, 72076 Tübingen, Germany.
}

\section{Authors' contributions}

$\mathrm{AB}$ carried out the $\mathrm{BCl}$ experiments for this study, adapted the $\mathrm{BCl}$ system to include the feedback bias, performed the statistical analysis and participated in the writing of the manuscript. MGW conceived and supervised the study, and participated in the data acquisition, statistical analysis and writing of the manuscript. All authors read and approved the final manuscript.

\section{Competing interests}

The authors declare that they have no competing interests.

Received: 10 December 2009 Accepted: 27 July 2010

Published: 27 July 2010

\section{References}

1. Wolpaw JR, Birbaumer N, Heetderks WJ, McFarland DJ, Peckham PH, Schalk G, Donchin E, Quatrano La, Robinson CJ, Vaughan TM: Brain- computer interface technology: a review of the first international meeting. IEEE Transactions on Rehabilitation Engineering 2000, 8:164-173.

2. Mason S, Bashashati A, Fatourechi M, Navarro K, Birch G: A comprehensive survey of brain interface technology designs. Annals of Biomedical Engineering 2007, 35(2):137-169.

3. Pfurtscheller G, Neuper C: Motor Imagery and Direct Brain-Computer Communication. Proceedings of the IEEE 2001, 89(7):1123-1134.

4. McFarland D, McCane L, Wolpaw J: EEG-based communication and control: short-term role of feedback. IEEE Transactions on Biomedical Engineering 1998, 6:7-11.

5. Neuper C, Scherer R, Wriessnegger S, Pfurtscheller G: Motor imagery and action observation: Modulation of sensorimotor brain rhythms during mental control of a brain-computer interface. Clinical Neurophysiology 2009, 120(2):239-247.

6. Curran EA, Stokes MJ: Learning to control brain activity: A review of the production and control of EEG components for driving brain-computer interface (BCl) systems. Brain and Cognition 2003, 51(3):326-336.

7. Grosse-Wentrup M, Liefhold C, Gramann K, Buss M: Beamforming in noninvasive Brain-Computer Interfaces. IEEE Transactions in Biomedical Engineering 2009, 56(4):1209-1219.

8. Ramoser H, Mueller-Gerking J, Pfurtscheller G: Optimal Spatial Filtering of Single Trial EEG During Imagined Hand Movement. IEEE Transactions on Rehabilitation Engineering 2000, 8(4):441-446.

9. Blankertz B, Sannellia C, Halder S, Hammer E, Kübler A, Müller KR, Curio G, Dickhaus T: Neurophysiological predictor of SMR-based BCl performance. Neurolmage 2010, 51(4):1303-1309.

10. Grosse-Wentrup M, Hill J, Schölkopf B: Causal Influence of Gamma Oscillations on the Sensorimotor-Rhythm. Neurolmage 2010.

doi:10.1186/1743-0003-7-34

Cite this article as: Barbero and Grosse-Wentrup: Biased feedback in brain-computer interfaces. Journal of NeuroEngineering and Rehabilitation 2010 7:34.

\section{Submit your next manuscript to BioMed Central and take full advantage of:}

- Convenient online submission

- Thorough peer review

- No space constraints or color figure charges

- Immediate publication on acceptance

- Inclusion in PubMed, CAS, Scopus and Google Scholar

- Research which is freely available for redistribution

Submit your manuscript at www.biomedcentral com/submit
C Biomed Central 